\title{
First results from DesertSTAR: a 7- pixel 345-GHz heterodyne array receiver for the Heinrich Hertz Telescope
}

Christopher E. Groppi, Christopher K. Walker, Craig Kulesa, Dathon Golish, Abigail Hedden, et al.

Christopher E. Groppi, Christopher K. Walker, Craig Kulesa, Dathon Golish, Abigail Hedden, Gopal Narayanan, Arthur W. Lichtenberger, Jacob W. Kooi, Urs U. Graf, Stefan Heyminck, "First results from DesertSTAR: a 7-pixel 345GHz heterodyne array receiver for the Heinrich Hertz Telescope," Proc. SPIE 5498, Millimeter and Submillimeter Detectors for Astronomy II, (8 October 2004); doi: 10.1117/12.552492

EDent: SPIE Astronomical Telescopes + Instrumentation, 2004, Glasgow, United Kingdom 


\title{
First Results from Desert STAR: a 7 pixel 345 GHz Heterodyne Array Receiver for the Heinrich Hertz Telescope
}

\author{
C.E. Groppi ${ }^{\mathrm{a}^{*}}$, C.K. Walker ${ }^{\mathrm{b}}$, C. Kulesa ${ }^{\mathrm{b}}$, D. Golish ${ }^{\mathrm{b}}$, A. Hedden ${ }^{\mathrm{b}}$, G. Narayanan ${ }^{\mathrm{c}}$, A.W. \\ Lichtenberger $^{\mathrm{d}}$, J. Kooi ${ }^{\mathrm{e}}$, U.U. Graf ${ }^{\mathrm{f}}$, S. Heyminck ${ }^{\mathrm{f}}$ \\ a) National Radio Astronomy Observatory; b)Steward Observatory, University of Arizona; \\ c)University of Massachusetts; d)University of Virginia; e)California Institute of Technology; \\ f)Universitat zu Köln
}

\begin{abstract}
We present the first astronomical results from DesertSTAR, a 7 pixel heterodyne array receiver designed for operation in the astrophysically rich $345 \mathrm{GHz}$ atmospheric window. DesertSTAR was constructed for the 10m Heinrich Hertz Telescope located at $3150 \mathrm{~m}$ elevation on Mt. Graham, Arizona. This receiver promises to increase mapping speed at the HHT by a factor of $\sim 15$ over the facility's existing single beam, dual polarization receiver. DesertSTAR uses tunerless, single-ended waveguide SIS mixers to achieve uncorrected receiver noise temperatures of $\sim 60 \mathrm{~K}$. The instantaneous bandwidth is $2 \mathrm{GHz}$, with a $5 \mathrm{GHz}$ Intermediate Frequency, offering $1600 \mathrm{~km} / \mathrm{s}$ of velocity coverage. Cryogenic isolators are employed between the mixers and low noise amplifiers to assure a flat IF passband. The system uses a Joule-Thompson closed-cycle refrigerator with $180 \mathrm{~W}$ capacity at $70 \mathrm{~K}$ and $1.8 \mathrm{~W}$ capacity at $4 \mathrm{~K}$. A novel reflective phase grating is used for Local Oscillator multiplexing, while a simple Mylar beamsplitter is used as an LO diplexer. Optics include only polyethelene mixer lenses and a single, cold, flat mirror, maximizing simplicity for high efficiency and easy optical alignment. The computer controlled bias system provides low noise bias for the SIS junctions, magnets and LNAs through a modular and hardware independent GUI interface, and allows remote operation and monitoring. We present measurements of receiver noise, beam quality, efficiency and stability in addition to astronomical observations obtained during engineering runs at the HHT.
\end{abstract}

Keywords: SIS Heterodyne Array Receiver Submillimeter Molecular Spectroscopy

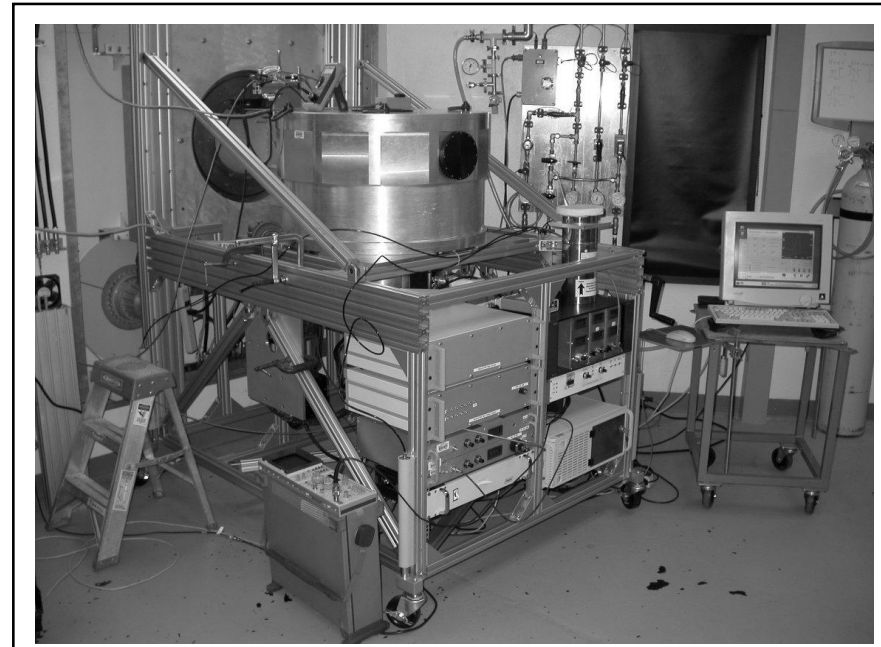

Figure 1: DesertStar mounted on the right Naysmith flange of the HHT during an engineering run in October, 2003.

\section{Introduction}

While the standard for heterodyne receivers at millimeter and submillimeter-wave telescopes around the world is still a single pixel, many multibeam receivers are currently in use (BEARS ${ }^{1}$, CHAMP $^{2}$ SMART $^{3}$, Pole$\mathrm{Star}^{4} \&$ HERA $^{5}$ and being developed (HARP-B ${ }^{6}$ ) Given that $\sim 55 \%$ of the time available at the HHT is suitable for $345 \mathrm{GHz}$ observations, we have concentrated our array development for this atmospheric window. Array receivers can dramatically increase the speed of mapping, making difficult projects easier and bringing impossible projects into reach. SCUBA and SHARC revolutionized submillimeter continuum observing. Heterodyne arrays have the same promise for high resolution spectroscopy. Heterodyne focal plane arrays with modern, tunerless mixers can increase observing efficiency in imaging mode by more than an order of magnitude over the previous generation of single pixel receivers with tuners. DesertStar will increase imaging

\footnotetext{
*cgroppi@ nrao.edu, phone 520-882-8250; fax 520-882-7955; http://www.tuc.nrao.edu/ cgroppi/; NRAO, 949 N. Cherry Ave, Tucson, AZ, USA 85721
} 
speed at the HHT by a factor of $\sim 15$ compared to the current MPIfR dual polarization $345 \mathrm{GHz}$ receiver. For many projects, like moderate spatial resolution Galactic plane CO surveys, this is enabling technology. Heterodyne arrays make the most efficient use of good weather, increasing the observing efficiency of single dish radio observatories.

\section{Instrument Description}

\subsection{Overview}

DesertSTAR was designed for use as a facility instrument for the Heinrich Hertz Telescope, located at 10,500 ft. on Mt. Graham in southeastern Arizona. The telescope is a 10m Cassegrain design with Carbon Fiber Reinforced Plastic (CFRP) primary reflector panels and backup structure. The quadrupod and the subreflector are also CFRP. The CFRP construction provides very good stiffness, plus the temperature stability necessary to operate 24 hours per day ${ }^{7}$. The surface of the main reflector has an RMS roughness of $\sim 13 \mu \mathrm{m}$, measured with holography. The HHT is currently the most accurate large radio telescope in existence. DesertSTAR will be mounted on the right Nasmyth platform, bolted directly to the telescope flange. Figure 1 is a photograph of DesertSTAR on the right Nasmyth flange of the HHT during an engineering run in October, 2003. The flange opening itself limits the maximum number of pixels. With the $2 \mathrm{~F} \lambda$ spacing of the pixels in DesertSTAR, the outer beams clear the flange at the $3 \omega$ level. The beams on the sky, as shown in Figure 2, are 22" FWHM, with 44 " center-to-center spacing, arranged in a close packed hexagonal

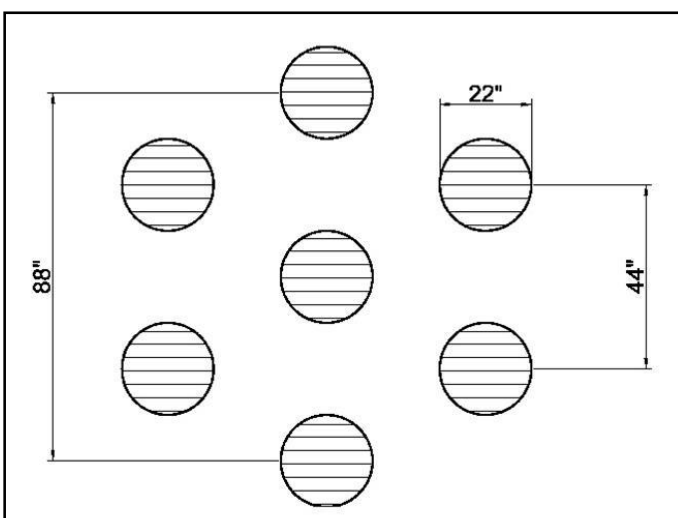

Figure 2: The FWHM beam footprint of DesertStar. Beams are 22" FWHM, separated by 2 beams (44"). The array span is $88^{\prime \prime}$. arrangement. This configuration maximizes the number of beams through the telescope flange on the HHT, while still preserving $\sim 20 \mathrm{~dB}$ isolation between pixels.

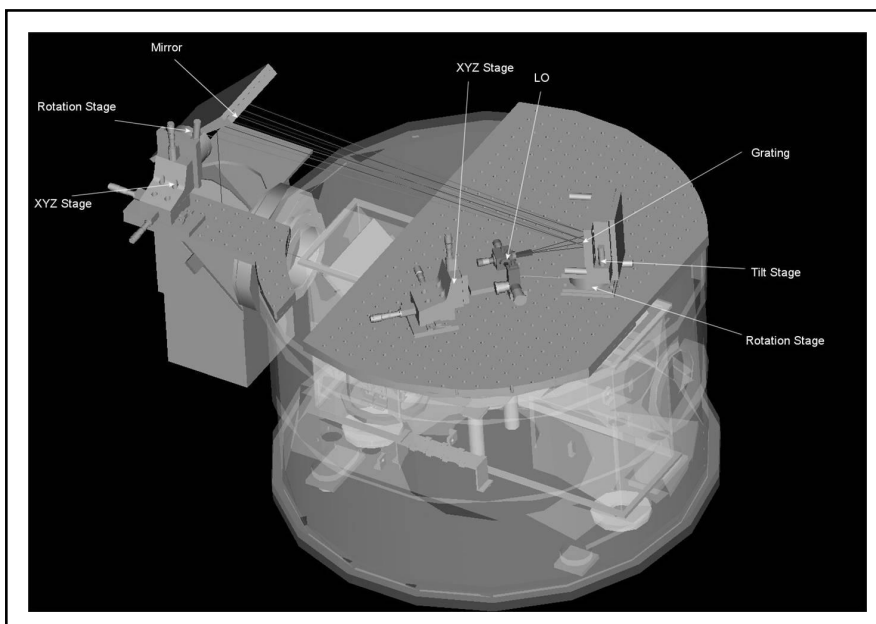

Figure 3: A transparent CAD rendition of DesertStar detailing the LO injection optics, cold optics and vacuum window configuration.
DesertSTAR uses fixed-backshort SuperconductorInsulator-Superconductor (SIS) mixers with a broadband waveguide probe. Instantaneous bandwidths of $2 \mathrm{GHz}$ can be achieved over the entire $345 \mathrm{GHz}$ atmospheric window. A cryostat with a JouleThompson (JT) mechanical refrigerator allows continuous operation and $1.8 \mathrm{~W}$ of cooling capacity at $4 \mathrm{~K}$, and provides the needed temperature stability for low-noise operation. Local Oscillator (LO) distribution is accomplished with a novel phase grating that yields high efficiency and power uniformity in a hexagonally symmetric geometry. The computer controlled bias system is an evolution of a proven design that is simple, portable to any computer platform, and readily extensible to over 100 channels. It provides bias control of the mixers, IF amplifiers and magnets as well as monitoring of temperature and vacuum. It can be operated from any X-windows capable machine and writes an instrument status web page visible with any web browser. The $2 \mathrm{GHz}$ Intermediate Frequency (IF) bandwidth allows the future addition of a wideband backend optimized for extragalactic observations, with $\sim 1700 \mathrm{~km} / \mathrm{s}$ of velocity coverage. 


\subsection{Optics}

Given that an array receiver is already a very complicated system, we chose to make the optics of DesertSTAR as simple as possible. Experience with the PoleSTAR ${ }^{4} 810 \mathrm{GHz}$ array at the AST/RO telescope on the South Pole has shown that in practice, complex optical circuits are difficult to implement with array receivers. An example is the Martin-Pupplet interferometer, which can be used as a quasi-optical LO diplexer or single sideband filter. Even a 0.5 degree misalignment of a beam propagating through the interferometer will cause a $3 \mathrm{~dB}$ loss in coupling. With an array architecture based on individual mixers mounted in the focal plane, it is difficult to control the beam boresight to the required tolerances. Other arrays, like $\mathrm{CHAMP}^{2}$ and $\mathrm{SMART}^{3}$ have gone through great pains to ensure alignment of optical components and mixers. We have chosen to eliminate all optics from the system that require extremely tight tolerances on mixer beam boresight, and minimize the number of lenses and mirrors. The beam from each diagonal horn is matched to the telescope with separate High Density Polyethylene (HDPE) lens mounted directly to the mixer housing, decreasing the chances of mixer squint due to horn-lens misalignment. The beams then reflect off a single, cold $45^{\circ}$ flat mirror and directly illuminate the flat tertiary of the telescope. Most designs use a curved mirror to cross the beam bundle at the location of the vacuum window to minimize window size and then use another curved mirror to parallelize expanding beams. We chose to keep the beams parallel throughout at the cost of increased vacuum window size.

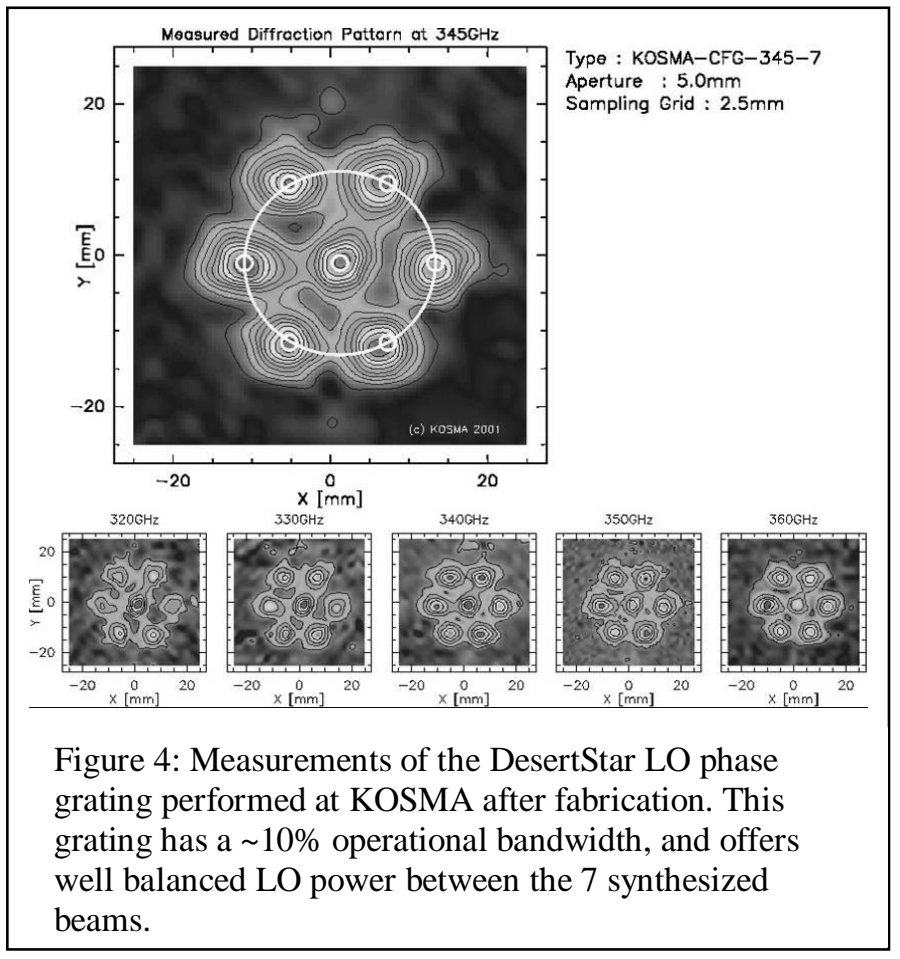

A new material, ZoteFoam PPA-30, made it possible to construct a 4.5 in. clear aperture vacuum window with low loss and good vacuum properties. ZoteFoam PPA30 is a closed-cell polyethylene foam blown with dry nitrogen gas. A 1" thick window 8" in diameter (1.75" glue surface, 4.5 " clear aperture) is sufficient to hold vacuum with no other materials present, with acceptable deflection under vacuum, and no noticeable helium permeability or outgassing problems. Performance from $200-400 \mathrm{GHz}$ is quite good, with losses of less than $0.02 \mathrm{~Np} / \mathrm{cm}$, but the loss rises rapidly at frequencies higher than $400 \mathrm{GHz}$. The loss saturates at $\sim 0.14 \mathrm{~Np} / \mathrm{cm}$ at $\sim 900 \mathrm{GHz}$. The noise contribution of the DesertStar window is between 5-10K. This is about twice the added noise of a 0.5 mil Mylar window or a Polyethylene coated crystalline quartz window. Zotefoam was chosen as the best option, since Mylar cannot span a window opening that large, and a quartz window would cost more than $\$ 8000$. It has been in use as a window material in an instrument at the South Pole (ACBAR) for several years with no reliability problems $^{8}$. We have been operating DesertStar with this vacuum window for almost two years with no reliability or vacuum hold time issues.

\subsection{Local Oscillator}

Local oscillator power delivery is another difficult problem for array receivers. LO power must be efficiently and equally divided into each pixel. While many waveguide techniques are available, they are complicated, difficult to implement and lossy at high frequencies. Quasi-optical techniques can also be lossy and complicated, with difficult alignment issues. Recent work by the Cologne and MPIfR groups in Germany have lead to the development of both transmissive and reflective phase gratings that form multiple LO beams from a single input beam through diffraction. Our LO distribution system uses a novel reflective grating design from the University of Cologne ${ }^{9}$. This design creates a 7 beam array with hexagonal symmetry with $80 \%$ efficiency and is superimposed on a parabolic mirror. This optic acts as a collimating mirror for the LO beam, and also forms the 7 beam array. The phase grating has an operational 
bandwidth of $\sim 10 \%$ centered on $345 \mathrm{GHz}$. The entire tuning range of the receiver can be covered with a few gratings that can be changed depending on receiver tuning. A simple Mylar beamsplitter acts as the LO diplexer. Figure 3 is a cutaway CAD drawing of the LO optics and receiver optics. Figure 4 shows the measured beam pattern of the LO phase grating at $345 \mathrm{GHz}$, with insets at other frequencies. This measurement was made at the University of Cologne by Urs Graf with a facility $350 \mathrm{GHz}$ receiver system.

\subsection{Cryogenics}

DesertSTAR was designed to allow for cooled optics and have room for a second sub-array. To achieve these goals, we designed a cryostat, with a 28 " cold plate, a large $4 \mathrm{~K}$ volume for cold optics, and a large $4 \mathrm{~K}$ cooling capacity. The cryocooler is based on the proven NRAO JT refrigerator, used for the facility receivers at the NRAO $12 \mathrm{~m}$ telescope. The unit has a $180 \mathrm{~W}$ capacity at $77 \mathrm{~K}$, a $1.8 \mathrm{~W}$ capacity at $4 \mathrm{~K}$, a $20 \mathrm{~K}$ helium precooler stage and a self-cleaning JT expansion valve. The refrigerator is mated to a commercial Balzers cold head, and driven by a custom NRAO compressor. Cooldown tests show stable $4 \mathrm{~K}$ operation is achieved in $\sim 13$ hours. In addition, we have used a resistive heater on the $4 \mathrm{~K}$ cold plate to measure a cooling capacity of $1.44 \mathrm{~W}$. This measurement was made with full infrared (IR) loading on the cold plate. Calculations show that the total heat load including amps and infrared loading is $0.9 \mathrm{~W}$. The IR heat load is dumped to the $77 \mathrm{~K}$ shield with a Gore-Tex GR IR filter. Since cryogenic testing began in 2000 , we have experienced no failures of the cryogenic system.

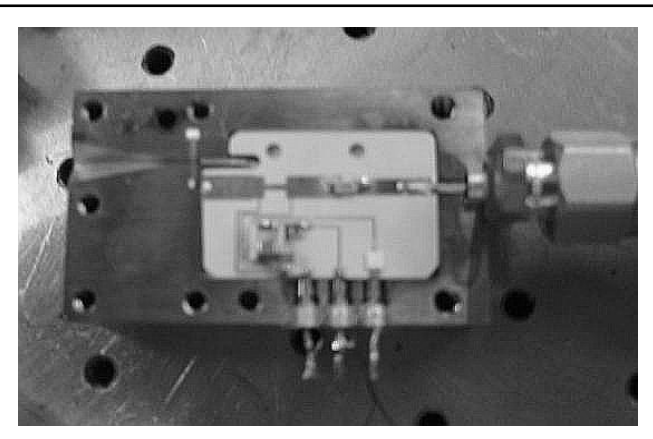

Figure 5: The single ended, fixed tuned DesertStar mixer block. Blocks are machined using the UMass micromilling system. The mixer includes an integrated bias tee.

\subsection{Mixers and Cold Electronics}

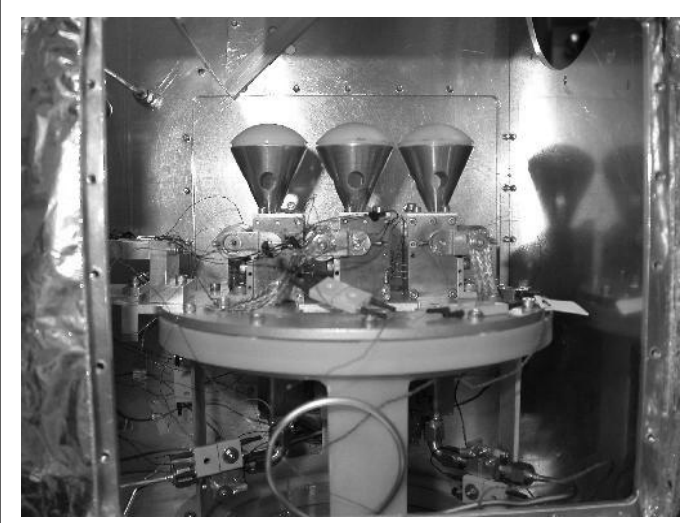

Figure 6: The DesertStar focal plane with the center row populated with three mixers.
DesertSTAR uses 7 independent single ended mixers, each mounted together with a HDPE lens, isolator and amplifier in a modular assembly, which is cooled by the JT refrigerator via a cold finger. The mixers were designed in collaboration with Gopal Narayanan at the University of Massachusetts and Jacob Kooi at Caltech. The mixer blocks were machined at UMass, and the SIS junctions fabricated at the University of Virginia by Art Lichtenberger. The simple, single ended waveguide design uses a diagonal feedhorn to couple the telescope beam through a multi section impedance transformer to half height waveguide. The waveguide backshort is fixed and there is no E-plane tuner. The cross-guide probe uses the proven suspended stripline design of Blundell \& Tong ${ }^{10}$. The Nb SIS junctions were designed with an on-chip tuning structure for low return loss across the atmospheric window, permitting a fixed backshort. Junction performance was simulated using the embedding impedance determined from scale model measurements, and assuming a $40-\mathrm{j} 20 \Omega$ embedding impedance, with similar qualitative results. The optimum backshort position was also determined via HFSS modeling. The diagonal horn was chosen since it could be readily made with the UMass micro-milling machine. While their coupling to the fundamental Gaussian mode is worse than scalar corrugated horns, diagonal horns still provide adequate broadband performance at a fraction of the $\operatorname{cost}^{11}$. The beam pattern is Gaussian, but the diagonal horn has about $14 \%$ crosspolarization, lowering its efficiency when used in combination with a linearly polarized input signal. Since DesertStar has no polarization defining optics before the mixer, the horn crosspolarization does not impact the main beam efficiency. A 4-wire bias tee is mounted in the mixer block, and monitors junction voltage and current with a precision current-sense resistor. Return loss is better than $-20 \mathrm{~dB}$ from $4-6 \mathrm{GHz}$ and leakage is less than $-40 \mathrm{~dB}$. Insertion 
loss is a fraction of a dB. The mixer produces a 4-6 GHz IF, and is connected via a Pamtech cryogenic ferrite isolator to a Miteq 4-6 GHz cryogenic low noise amplifier (LNA). Losses of the bias tee and isolator are less than 1.5dB from 4-6 $\mathrm{GHz}$, as measured with a vector network analyzer. The Miteq amplifiers have $27 \mathrm{~dB}$ gain, less than $1 \mathrm{~dB}$ passband ripple, $30 \mathrm{~mW}$ power dissipation and a measured noise temperature of $7 \mathrm{~K}$ at $4 \mathrm{~K}$ operating temperature. The amplifiers require only a single, unipolar $0.6 \mathrm{~V}$ bias, greatly simplifying the wiring and bias supplies. A simple superconducting coil electromagnet provides the necessary magnetic flux to minimize Josephson noise with soft iron field concentrators embedded in the mixer block. Figure 5 is a closeup photograph of a prototype mixer, showing the diagonal horn, waveguide and junction channel. Figure 6 shows the mixer array inside the cryostat with 3 mixers installed.

\subsection{Electronics}

Array receivers present a new challenge to the DC bias electronics necessary to provide power to the SIS junctions, magnets and LNAs. Simple boxes with manual controls are far too cumbersome to implement with a large number of pixels. To solve this problem, we have developed a computer controlled bias system. The electronics are based on the design used with single pixel receivers at the HHT, but replace manual potentiometers with digital potentiometers that communicate over the Philips $\mathrm{I}^{2} \mathrm{C}$ bus. Each bias card contains 4 channels of electronics. The DesertSTAR system uses two SIS bias cards. The LNA and magnet bias system each use 4 cards. Two analog multiplexer cards select monitor signals. The SIS bias cards include low impedance solid state switches to disengage the constant voltage servo circuit for fast I-V curve sweeps. Both the $\mathrm{I}^{2} \mathrm{C}$ input from the control computer and the DC monitor voltage outputs are optically isolated, eliminating any electrical connection between the bias rack and computer. A simple data-IO card in the computer digitizes the monitor signals. The Linux control computer runs a graphical interface written in C/GTK+, which provides a user-friendly interface to the bias parameters. Cryogenic, vacuum and LO status are also available on-screen. The $\mathrm{X}$-windows interface allows the receiver to be optimized from any $\mathrm{X}$-windows capable machine, which will allow the instrument builders to diagnose and fix tuning problems remotely. The software has been written in a hardware independent manner, allowing easy hardware changes with a minimum of new code development.

\subsection{Backends}

The HHT is currently upgrading the telescope control system and backend electronics for all receivers. As a part of this effort, a modern, flexible filterbank spectrometer with a $2 \mathrm{GHz}$ bandwidth and $1 \mathrm{MHz}$ resolution is being constructed. The IF processor for this backend can support $8 \times 256$ $\mathrm{MHz}$ IF sub-bands for use with DesertSTAR. This bandwidth and velocity resolution is adequate for a large variety galactic astronomy projects. The filterbank should provide extremely high stability without the platforming problems often associated with hybrid correlators. Figure 7 shows the HHT FBS system under construction in the lab. In the future,
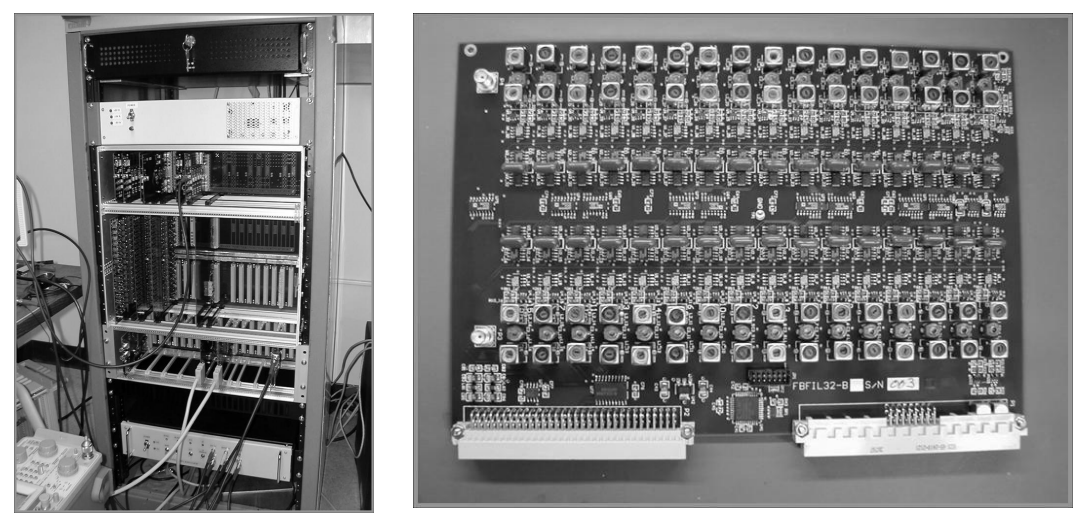

Figure 7: The HHT Filter Bank Spectrometer system under construction (left) and a prototype filter card (right). A flexible IF processor will allow configuration of the FBS as a $8 \times 256 \mathrm{MHz}$ array spectrometer. we hope to obtain a 7 channel digital autocorrelator with $2 \mathrm{GHz}$ of IF bandwidth per channel. This will allow extragalactic observing with the full $2 \mathrm{GHz}$ IF bandwidth of DesertSTAR with $8 \mathrm{MHz}$ resolution. The flexibility of the correlator also allows for widely variable resolution via an adjustable system clock. During the 2003 season, we operated the receiver in three pixel mode using the existing facility acousto-optical spectrometers. 


\section{Receiver Performance}

\subsection{Preliminary Results}

Initial tests of a single array mixer/IF chain were conducted in an IR Laboratories $\mathrm{LN}_{2} /{ }^{4} \mathrm{He}$ cryostat. The test system uses a 0.5 mil Mylar LO diplexer, a 1.0 mil Mylar cryostat vacuum window, and a single layer of Zitex-A for IR filtering. The mixer beam is launched with an unmatched HDPE lens mounted directly to the mixer housing. The first prototype mixer has a very long backshort channel that is shortened to the proper length with aluminum shim stock. This allows some adjustability of the backshort position. All other mixers were fabricated with a machined, non-adjustable backshort. A room temperature total power box and a Hewlett-Packard power detector were used for the Yfactor measurements. The bias control system interrogates the output of this meter to allow total power vs. bias voltage sweeps. Before mounting a device in the mixer block, we tested 20 devices using a dipstick inserted into a liquid helium storage dewar. Of the $\sim 20$ devices tested, only 3 were nonfunctional, and the remaining devices appeared to be virtually identical. The device mounted in the test block was randomly selected from the good devices. We are currently evaluating the two tuning circuit designs and junction sizes. Tests so far from 320-

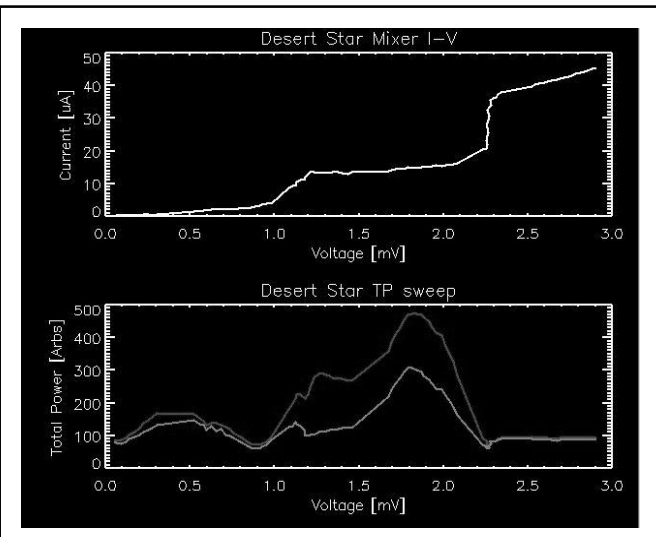

Figure 8: Pumped IV curve and hot/cold total power sweeps for a representative DesertStar mixer.

$360 \mathrm{GHz}$ show little or no difference in performance between the tested devices. We expect the differences to become apparent at the edges of the receiver band, but we are currently limited by our LO tuning range.

\section{2. $\quad$ Test Receiver Performance}

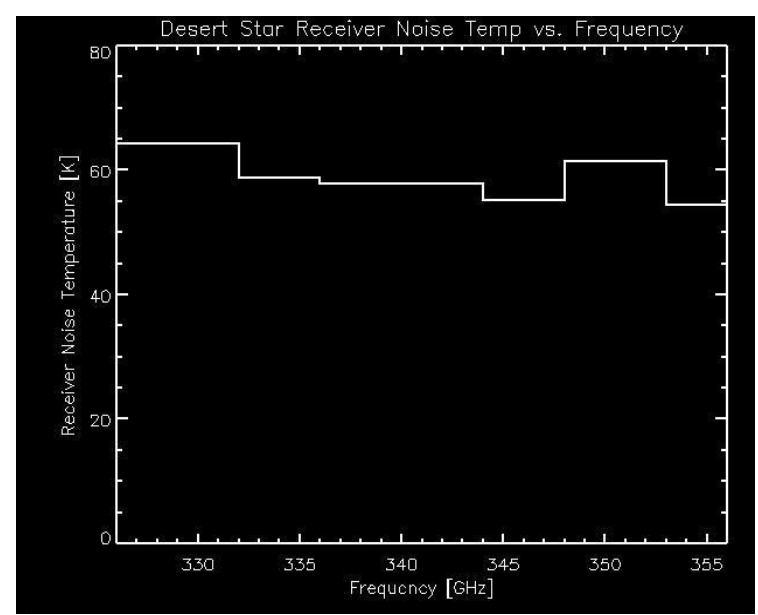

Figure 9: Uncorrected receiver noise temperature for a representative DesertStar mixer. Tuning range is limited by the bandwidth of the LO chain.
Figure 8 shows a pumped I-V curve of the test mixer, along with hot and cold total power curves. The bias system uses the slope of the supercurrent at $0 \mathrm{~V}$ to measure the contact resistance, and then remove it from the I-V curve. We measured $\mathrm{Rn} 48.5 \Omega$, a leakage current of $\sim 3 \mu \mathrm{A}$ and a transition voltage of $\sim 2.3 \mathrm{mV}$. Optimum bias voltage for the highest Y-factor was $\sim 1.6 \mathrm{mV}$. We expect $\mathrm{T}_{\mathrm{rx}}$ to remain fairly constant from $\sim 300 \mathrm{GHz}$ to $\sim 390 \mathrm{GHz}$, from the HFSS and Supermix simulations of the mixer return loss. Measurements indicate receiver temperatures of $\sim 55 \mathrm{~K}$ across the available band, with fairly flat response, as shown in figure 10. These are uncorrected noise temperatures, and include the losses of the LO diplexer, window and unmatched lens, along with noise from the IF chain. At this bias point, we measured total power stability to be better than $0.5 \%$ over 10 minutes. Y factor measurements using a spectrum analyzer show the $\mathrm{Y}$ factor is essentially constant across the 4-6 GHz IF. The LO source used in the test was unable to tune below $326 \mathrm{GHz}$ or above $356 \mathrm{GHz}$, so we could not measure the receiver temperature over the full band of the waveguide probe. 


\subsection{Instrument Performance Characterization}

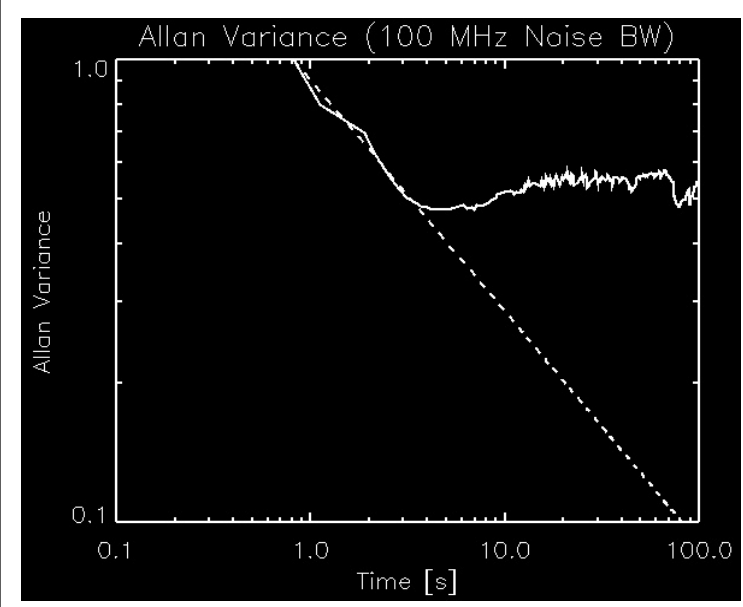

Figure 10: Measurement of Allan variance for a DesertStar mixer. This measurement was performed using the full $2 \mathrm{GHz}$ IF bandwidth, then scaled to a $100 \mathrm{MHz}$ detection bandwidth assuming a $\mathrm{t}^{2}$ power law noise spectrum.
After characterizing the performance of a single mixer in the test system, we installed the prototype mixer, and two additional mixers with fixed, machined backshorts into the JT cryostat. We repeated the same tests done in the test setup, and found that the mixers behaved identically, but with receiver noise temperatures of $\sim 60 \mathrm{~K}$. It is not uncommon for the noise of a mixer in an array dewar to be slightly higher than in a highly optimized single pixel test system. We believe that losses in the cryostat window material and IF losses in the long runs of semi-rigid coaxial cable are responsible for the increase in noise temperature. We measured the loss of the window material by inserting a slab of ZoteFoam in the beam and observing the change in Y-factor. We found that the ZoteFoam contributed $\sim 5 \mathrm{~K}$ to the receiver noise temperature.

\subsubsection{Stability Analysis}

We performed an Allan variance stability analysis of the receiver to determine if microphonics from the large Balzers cryo-head would negatively effect receiver stability. We used a Hewlett-Packard power meter, sampled every $50 \mathrm{~ms}$ with a data acquisition card in the computer control system. Data was taken

for more than 1.5 hours, and folded every 5 minutes to lower the noise due to the short integration time. We measured the total power over the entire 4-6 GHz IF for this test, and then scale the result to a $100 \mathrm{MHz}$ postdetection bandwidth, since we did not have a narrow-band $5 \mathrm{GHz}$ center frequency filter on hand. The data were analyzed following Kooi et al. $2000^{12}$. We assume a power law noise spectrum proportional to $t^{2}$. Then the Allan time scales like $B^{-1 / 2}$. The results in Figure 10 show an Allen variance time of $\sim 3 \mathrm{~s}$ for a $100 \mathrm{MHz}$ bandwidth. This translates to an Allan variance time of $\sim 30$ s with a $1 \mathrm{MHz}$ post detection bandwidth. This stability time is adequate for all observing modes at the HHT. At the telescope, we limit integration times for position switched observations to less than 10s, since scaling the Allan time over this large a range is probably not fully valid.

\subsubsection{Beam Pattern Measurement}

Using a computer controlled XY stage system, we measured E and H plane cuts of the receiver beam pattern. The source was a liquid nitrogen cold load behind a sheet of room temperature absorber. A small hole in the absorber created a strong, negative source which could be detected using a lock-in amplifier and room temperature chopper wheel. The $3 \mathrm{~cm}$ source size allowed adequate sampling of the receiver beam, and provided sufficient signal. Several scans were coadded and then fit with a 5 component Gaussian. Results showed the beam to be Gaussian in shape, but the beam was more broad and elliptical than expected. The horn lenses were designed based upon the analytical prediction of the horn's emergent beam ${ }^{11}$. However, our later HFSS simulations show the actual horn beam to be wider than predicted. The over-illumination of the lens results in a emergent beam $15 \%$ too broad, in addition to cold loss from spillover onto the lens holder. We redesigned the lens mounts to compensate for this problem, and have confirmed the correct beam pattern with both lab

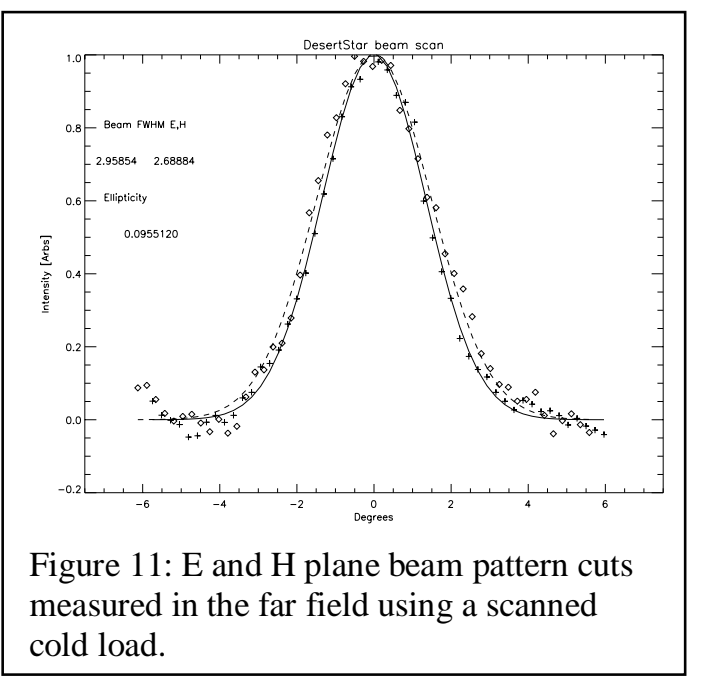


measurements, and measurements on the sky using Mars. Figure 11 shows the E and $\mathrm{H}$ plane cut data as well as the fitted beam patterns.

\section{Telescope Performance and Initial Results}

In early June, 2003 DesertStar was taken to the HHT for a 6 day engineering run. The goal was to identify areas of difficulty in using the receiver on the telescope, and to perform as many performance tests and verifications of the central mixer as possible. Overall, the results were good with some minor disappointments. Initial installation and cooldown were trouble free, but the receiver's mount proved to be inadequate. The weight of the receiver caused the mount to deform under load, and it was obvious that the mount was not rigid enough to prevent the receiver oscillating due to the cold-head cycle. The mount was reinforced as a stop-gap measure, and we continued to align the receiver to the telescope. Within two days, we had the central beam of the array on the sky, and had easily achieved receiver noise temperatures of $\sim 90 \mathrm{~K}$. Measurements with a laser level proved the cryostat was indeed oscillating with a peak-to-peak amplitude of more than 40." This oscillation prevented us from making fine pointing or focus measurements. We used spectra and maps from extended regions to coarsely point the telescope. On centrally concentrated sources, peak line temperatures were diluted since the receiver beam was not on-source for all the integration time. Spectra were obtained from a variety of sources including IRAS16293, IRC+10216, DR21, CepA and S140. This data was used to verify the proper operation of the receiver system. We were able to measure a moon efficiency of $\sim 80 \%$. This number is abnormally low due to the broader than expected beam from the diagonal feedhorns as mentioned in the previous section.

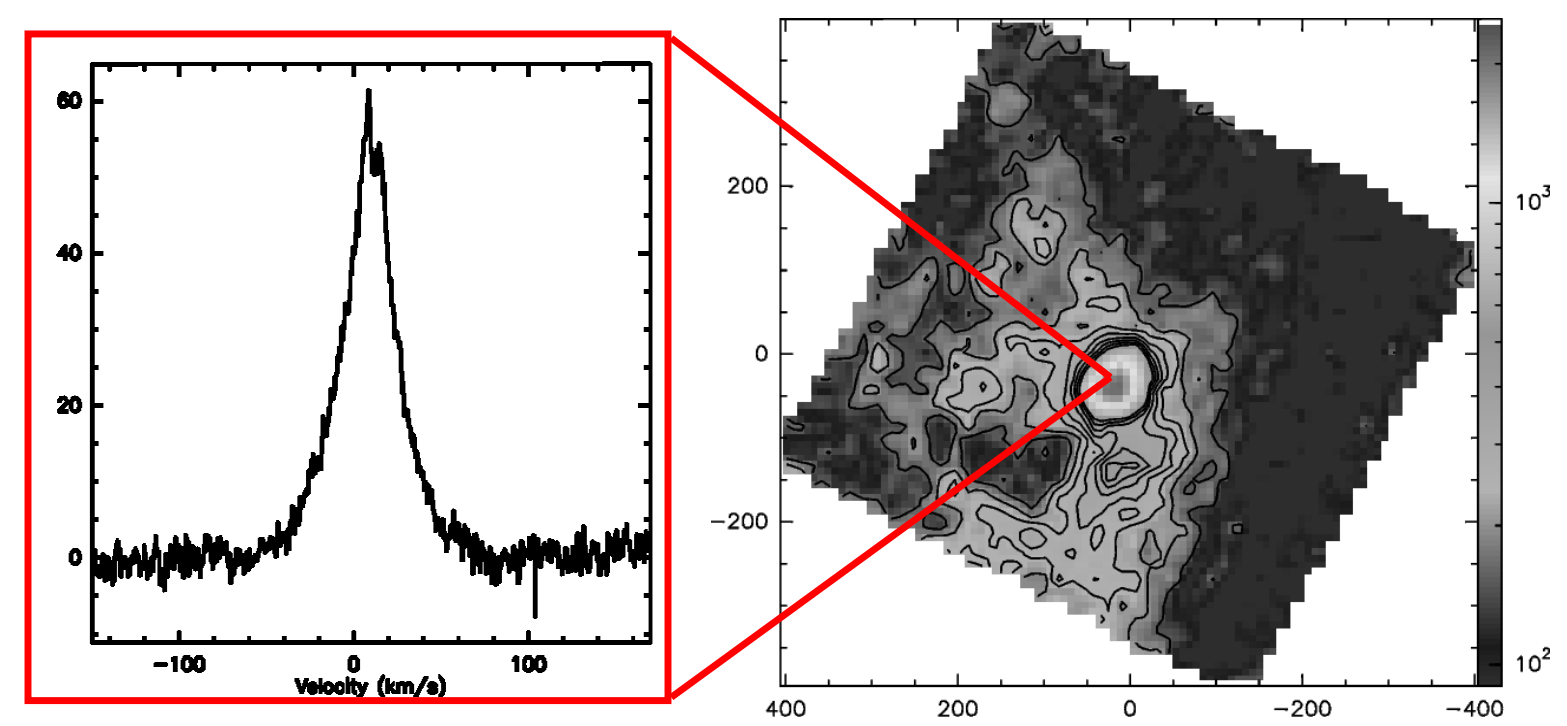

Figure 12: A 10x10 arcminute map of Orion IRC2 taken with DesertStar during the October, 2003 engineering run.

In October, 2003 DesertStar returned to the telescope with many of the previous problems fixed. A new mount (shown in figure 1) proved to be a great improvement over the previous design. In addition, we were able to simultaneously operate three mixers, although limitations in the telescope IF system, and a small optical misalignment only allowed us to observe with two mixers simultaneously. We did observe with all three mixers pairwise during the run and were able to achieve receiver noise temperatures of $\sim 60 \mathrm{~K}$ at $350 \mathrm{GHz}$ LO frequency. Measurements of main beam efficiency on Mars showed $\eta_{m b} \sim 0.80 \pm 0.05$ for the three mixers in the cryostat. Allan variance measurements repeated at the telescope confirmed the lab results. In addition we obtained several ${ }^{12} \mathrm{CO}(3-2)$ maps of Orion IRC2, DR21 and several other sources in OTF, beam switched and position switched observing modes. In April 2004, we staged a third engineering run with the goal of simultaneously observing with all three mixers, using software de-rotation of the outer beams. While the weather for this run was particularly poor, we were able to achieve the goal, and obtain maps of Orion IRC2 
simultaneously observing with all three mixers. Figure 12 shows a 10x10 arcminute map of Orion IRC2 taken during the October, 2003 run, with a representative spectrum. The RMS noise of this map is lower than that of a map derived from a single beam by the square root of the number of pixels, as expected.

\section{Upgrades}

In the summer of 2004, we will complete DesertStar, and implement several upgrades. Four additional mixers will be added to complete the focal plane. These mixers have been partially completed and will be delivered by UMass in the summer of 2004. In addition, we will mount the phase grating based LO injection system. This system has already been tested in the lab, and was able to pump a mixer using a $100 \%$ reflective LO diplexer. To use this system to pump all 7 mixers, we will fabricate a new mixer mounting plate to remedy a fabrication error. In addition, we will purchase a Virginia Diodes synthesizer driven LO chain capable of providing more than $1 \mathrm{~mW}$ of power from $310-380$ $\mathrm{GHz}$, with $\sim 4-5 \mathrm{~mW}$ of power available at the center of the band. This LO provides at least 10 times the LO power available from our current whisker contacted quadrupler driven with an $85 \mathrm{GHz}$ Gunn oscillator. The older LO source is capable of pumping three mixers using a simple dielectric sheet based LO power splitter.

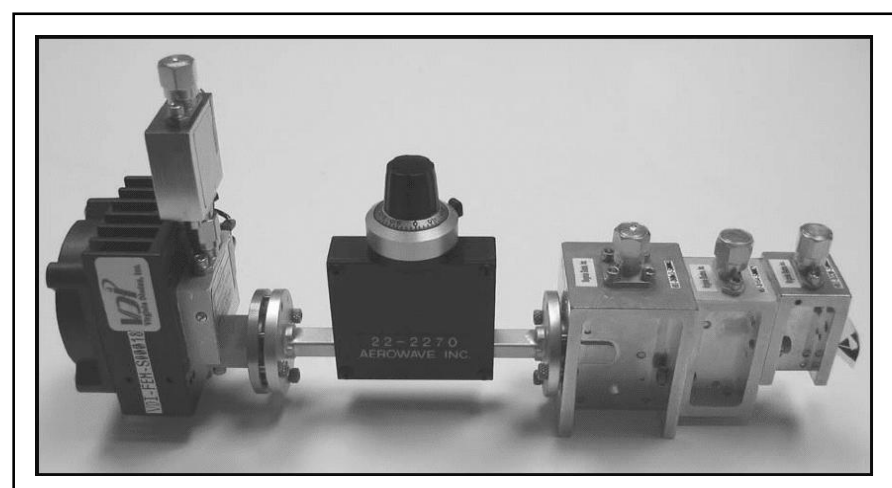

Figure 13: A Virginia Diodes synthesizer driven LO chain. Input is from a phase-locked synthesizer at $\sim 13$ $\mathrm{GHz}$. This signal is tripled and amplified, and followed by three doublers. Power output is greater than $1 \mathrm{~mW}$ from 310-380 GHz, with $4-5 \mathrm{~mW}$ at band center.

The new LO source will allow us to simultaneously pump all 7 mixers with the KOSMA phase grating, and the wider bandwidth will allow more exhaustive mixer testing. An image of a similar LO chain is shown in figure 13.

\section{Summary}

We have completed the first phase of constructing and testing a $345 \mathrm{GHz}$ heterodyne array receiver for use on the Heinrich Hertz Telescope on Mt. Graham in Arizona. The instrument uses fixed backshort, suspended stripline SIS mixers with $2 \mathrm{GHz}$ IF bandwidth. Cooling is provided by a high capacity closed cycle refrigerator in a large, expandable cryostat. We have demonstrated proper operation of the cryogenic system and computer controlled bias system. Measurements indicate the prototype array mixer can provide uncorrected receiver noise temperatures of $\sim 60 \mathrm{~K}$ from 326-356 GHz. Performance has been verified in the JT cryostat with 3 mixers, and the instrument has had three successful engineering runs on the HHT in June, 2003, October 2003 and April 2004. Receiver noise temperature, stability, optical performance and receiver reliability have all been verified at the telescope. The remaining 4 mixers will be added to the system in the summer of 2004, bringing the array up to its full compliment of 7 pixels. DesertStar is the first operational $345 \mathrm{GHz}$ array receiver. 


\section{BIBLIOGRAPHY}

1. K. Sunada, C. Yamaguchi, N. Kuno \& N. Ukita, Development and First Results of NRO 25-BEam Array Reciever System (BEARS), Imaging at Radio Through Submillimeter Wavelengths, ASP Conference Proceedings Series, Vol. CS-217, 2000.

2. R.. Gusten, First Results with CHAMP, the Carbon Heterodyne Array of the MPIfR, Imaging at Radio Through Submillimeter Wavelengths, ASP Conference Proceedings Series, Vol. CS-217, 2000.

3. Graf, U. U., Haas, S., Honingh, C. E., Jacobs, K., Schieder, R., Stutzki, J., Array receiver development at KOSMA for the submillimeter and terahertz spectral range, Proc. SPIE Vol. 3357, 1998.

4. Groppi, C.E. et al., Pole STAR: An $810 \mathrm{GHz}$ Array Receiver for AST/RO, Imaging at Radio Through Submillimeter Wavelengths, ASP Conference Proceedings Series, Vol. CS-217, 2000.

5. K. Schuster, J. Blondel, M. Carter, B. Fouilleux, B. Lazareff, M. Mattiocco, J. Pollet, The IRAM $230 \mathrm{GHz}$ Multibeam SIS Receiver, Imaging at Radio Through Submillimeter Wavelengths, ASP Conference Proceedings Series, Vol. CS-217, 2000.

6. W.R.F. Dent, HARP \& ACSIS on the JCMT, Imaging at Radio Through Submillimeter Wavelengths, ASP Conference Proceedings Series, Vol. CS-217, 2000.

7. Baars, J. W. M., Martin, R.N., Mangum, J.G., McMullin, J.P., Peters, W.L. The Heinrich Hertz Telescope and the Submillimeter Telescope Observatory, PASP, Volume 111, Issue 759, 1999.

8. Kuo, C.L. et al., ACBAR Measurements of CMB Angular Power Spectrum, American Astronomical Society Meeting 200, \#06.03, 2002.

9. Heyminck, S. \& Graf, U.U., Reflection gratings as THz local oscillator multiplexer, Proc. SPIE Vol. 4014, 1998.

10. Blundell, R., Tong, C-Y.E., Papa, D.C., Leombruno, R.L., Zhang, X., Paine, S., Stern, J.A., LeDuc, H.G. \& Bumble, B., Wideband fixed-tuned SIS receiver for 200-GHz operation, IEEE MTT Symp. on Space Terahertz Technology, Vol. 43, 1995.

11. Johansson, J.F., \& Whyborn, N.D., The Diagonal Horn as a Sub-Millimeter Wave Antenna, IEEE MTT, Vol. 40, No, 5, 1992.

12. Kooi, J.W., Chattopadhyay, G., Thielman, M., Phillips, T.G., \& Schieder, R. Noise Stability of SIS Receivers, Int J. IR and MM Waves, Vol. 21, No. 5, May, 2000. 